University of Nebraska - Lincoln

DigitalCommons@University of Nebraska - Lincoln

Norman R. Simon Papers

Research Papers in Physics and Astronomy

8-1-1989

On The Masses And Luminosities Of RR Lyrae Stars

Norman R. Simon

University of Nebraska - Lincoln, nsimon@unl.edu

Follow this and additional works at: https://digitalcommons.unl.edu/physicssimon

Simon, Norman R., "On The Masses And Luminosities Of RR Lyrae Stars" (1989). Norman R. Simon Papers. 29.

https://digitalcommons.unl.edu/physicssimon/29

This Article is brought to you for free and open access by the Research Papers in Physics and Astronomy at DigitalCommons@University of Nebraska - Lincoln. It has been accepted for inclusion in Norman R. Simon Papers by an authorized administrator of DigitalCommons@University of Nebraska - Lincoln. 
The Astrophysical Journal, 343:L17-L20, 1989 August 1

(C) 1989. The American Astronomical Society. All rights reserved. Printed in U.S.A.

\title{
ON THE MASSES AND LUMINOSITIES OF RR LYRAE STARS
}

\author{
NORMAN R. SIMON \\ Department of Physics and Astronomy, University of Nebraska-Lincoln \\ Received 1989 April 18; accepted 1989 May 17
}

\begin{abstract}
Some results are given from the comparison with observations of a large number of hydrodynamic pulsation models: (1) Linear pulsation calculations are adequate for the determination of the masses of RRd stars; (2) the Fourier phase parameter $\phi_{31}$ measures the luminosity-to-mass ratio $L / M^{1.81}$ for $R R c$ stars; (3) most of the $\omega$ Cen RRc stars seem to be evolved, as has previously been suggested; (4) the masses of the $\omega$ Cen RR $c$ stars agree with the masses of RRd stars in other clusters; and (5) a hint emerges of a mass-helium abundance relation among the $R R c$ stars in $\omega$ Cen. New CCD observations will be needed to extend our analysis to additional globular clusters.

Subject headings: stars: individual $(\omega$ Cen $)$ - stars: pulsation - stars: RR Lyrae
\end{abstract}

\section{INTRODUCTION}

RR Lyrae stars play an important role in our attempt to understand stellar evolution, the morphology and history of the Galaxy, and the cosmic distance scale. These stars have been studied much, with often conflicting and controversial results. Up to now, there is no general agreement on their masses and luminosities, certainly not at the level that would allow substantial progress on the problems mentioned above.

In the present Letter we describe some consequences emerging from the comparison with observations of a large grid of hydrodynamic pulsation models. For the sake of brevity, we shall present a minimal bibliography here, referring to review papers whenever possible. Details of the pulsation models will be published elsewhere, along with background, extensive references and additional ramifications of our results.

\section{PERIODS OF THE RR $d$ STARS}

The RR $d$ stars pulsate simultaneously in the first overtone and fundamental modes. These objects are found in certain globular clusters and dwarf spheroidal galaxies, and in the field (a single case). Their pulsation periods and period ratios, $P_{1} / P_{0}$, have been carefully monitored (e.g., Clement et al. 1986 and references therein). When these observed periods are compared with those emerging from linear nonadiabatic pulsation models, masses are obtained for the RR $d$ stars, as follows: Oo I clusters, $M \sim 0.55 M_{\odot}$; Oo II clusters, $M \sim 0.65 M_{\odot}(\mathrm{Cox}$ 1988 and references therein).

However, the above results depend on the ability of the models to reproduce observed period ratios with an accuracy $\sim 0.001$ ! Given this, it may reasonably be objected that the true theoretical periods, which is to say the nonlinear periods, may not agree with the linear values to the degree required. Such an objection is strengthened by many models in the literature for which the linear and hydrodynamic periods diverge by considerably more than the allowed amount (e.g., Simon and Aikawa 1986).

Because the disagreement between linear and nonlinear calculations could be due to the failure to allow the hydrodynamic models enough integration time to settle down, we have constructed two new models (one with the "canonical" parameters for Oo I RRd stars and the other for Oo II stars), and, in each model, separately integrated the fundamental and first- overtone modes for well over 1000 periods. This was sufficient for the maximum kinetic energy (evaluated over intervals of 10 periods) to settle down to one part in $10^{3}$. After this convergence had occurred, the periods were carefully measured. It was found that the period ratios obtained from these periods differed from the linear period ratios by $<0.001$. In view of the fact that a similar result was obtained by Kovacs and Buchler (1988) with a different code and a model with much different parameters, it may be concluded that no evidence exists that the use of linear models to infer $\mathrm{RRd}$ masses produces any error that is not negligible. Thus this objection to the $\mathrm{RR} d$ masses is removed, although the objection that the hydrodynamic models cannot produce sustained double-mode pulsation with reasonable parameters remains (e.g., Kovacs and Buchler 1988).

\section{THE PHASE PARAMETER $\phi_{31}$}

The observed light curves of pulsating stars may be compared with light curves from hydrodynamic models using the quantitative technique of Fourier decomposition. A recent review of this technique was given by Simon (1988a). In brief, one fits both the observed and theoretical light curves with a Fourier series of the form $A_{0}+\sum_{j} A_{j} \cos \left(j \omega t+\phi_{j}\right)$ and makes the comparison between observations and theory using combinations of the Fourier coefficients, viz., $R_{j 1}=A_{j} / A_{1}$, $\phi_{j 1}=\phi_{j}-j \phi_{1}$.

The hydrodynamic calculations have a mixed record in reproducing observed RR Lyrae light curves. Simon (1985) tested a small sample of hydrodynamic models and found that while the fundamental mode calculations failed to match observed Fourier parameters for the RRab stars, the firstovertone models reproduced the $\mathrm{RR} c$ observations rather well. Given this result, it was decided to construct a large grid of overtone calculations to attempt a detailed comparison with the $R \mathbf{R} c$ pulsators.

Forty-two models were integrated, with parameters within the limits $0.50 \leq M / M_{\odot} \leq 0.70,1.54 \leq \log L / L_{\odot} \leq 1.86$ and $6850 \leq T_{e} \leq 7500 \mathrm{~K}$. Two values of $Z$ were used $(0.001$ and $0.0002)$ and two values of $Y(0.25$ and 0.30$)$. While the metallicity had negligible effect on the pulsation properties of the models, the helium abundance was, as expected, much more important. For given $M, L$, and $Y$, two models were calculated, 
one near the overtone blue edge and one just redward $(<100 \mathrm{~K})$ of the fundamental blue edge.

Details regarding the calculations along with many other results will be published elsewhere. Here we focus on the Fourier phase parameter $\phi_{31}=\phi_{3}-3 \phi_{1}$. The 42 hydrodynamic models were fitted by least squares, yielding the expression

$\log L=0.105 \phi_{31}+1.814 \log M-0.081 \log Y+1.692$,

where $L, M$, and $Y$ are model parameters and $\phi_{31}$ was extracted from Fourier decompositions of the theoretical light curves. The standard deviation of this fit is only 0.03 in $\log L$ and the helium dependence is weak, indicating that $\phi_{31}$ is essentially measuring $L / M^{1.81}$. We now make a further fit to the model data, introducing a new variable, namely the period. We obtain (with standard deviation 0.36 )

$$
\phi_{31}=6.013 \log P-7.398 \log M+5.098 \log Y+7.571 \text {. }
$$

Now, $\phi_{31}$ and $\log P$ are both directly observable. Figure 1 shows $\phi_{31}$ versus period for a group of $\mathrm{RR} c$ stars in the globular cluster $\omega$ Centauri (Petersen 1984 and references therein) and in the field (Simon $1988 b$ and references therein). It can be seen that a large majority of the stars follow a relation of increasing $\phi_{31}$ with period. Note the clump of eight field stars at the lower left, with the remaining six field stars at longer period and larger $\phi_{31}$. Given that $\phi_{31}$ measures the luminosity to mass ratio as indicated above, then a reasonable interpretation of Figure 1 is that the clump of eight stars are zero-age horizontal-branch objects and the others are evolved. If this is true then Figure 1 also shows that the majority of the $\omega$ Cen $\mathrm{RR} c$ stars are themselves evolved as has previously been suggested by others (e.g., Dickens 1989 and references therein).

In Figure 2 we replot $\phi_{31}$ versus $P$ for a reduced sample, eliminating nine $\omega$ Cen stars that depart greatly from the trend

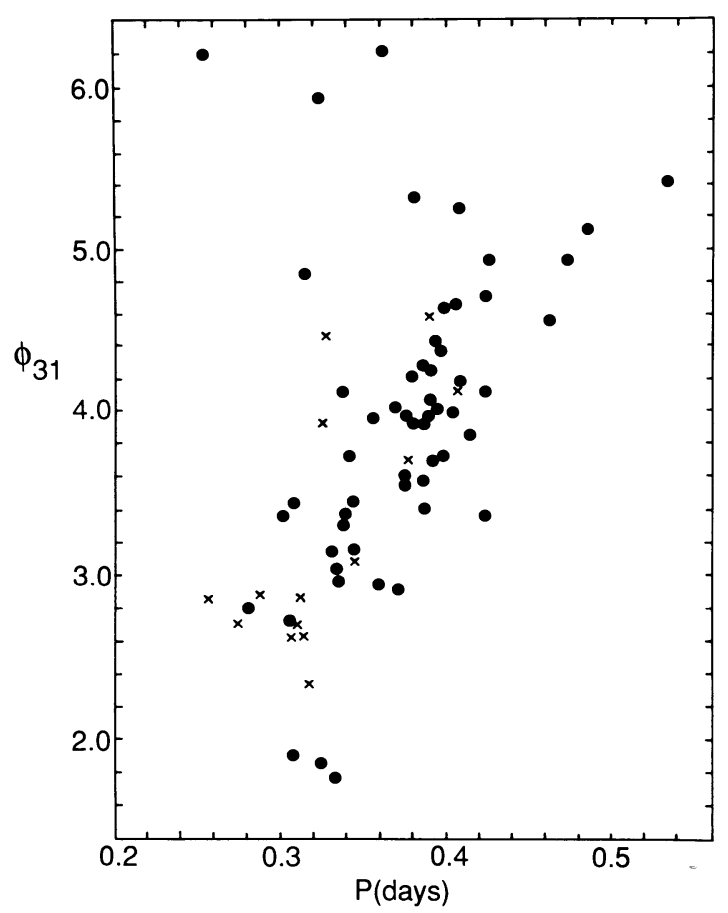

Fig. 1.-Values of $\phi_{31}$ vs. period for RR $c$ stars in $\omega$ Cen (dots) and in the field (crosses).

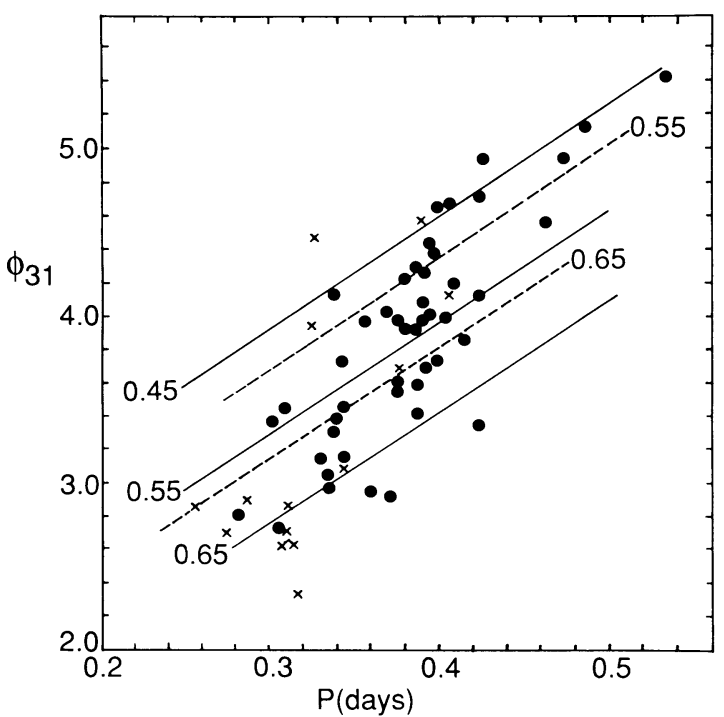

FIG. 2.-Same as Fig. 1 but with reduced sample for $\omega$ Cen. Lines represent theoretical loci from eq. (2), with masses indicated. Solid lines: $Y=0.25$, dashed lines: $Y=0.30$

in Figure 1. These objects may be anomalous due to observational error or may be truly eccentric (and thus of considerable interest), but we do not treat them here. Superposed on the observed points are theoretical loci taken from equation (2) for different masses and two helium abundances, $Y=0.25$ (solid lines) and $Y=0.30$ (dashed lines).

As a prelude to discussing Figure 2 we note the strong helium dependence in equation (2). This comes about because the period is very sensitive to the temperature of the model whereas $\phi_{31}$ is not. Since the model temperatures chosen for our study were determined by the pulsational blue edges whose location is, in turn, influenced by $Y$, the helium abundance has a significant effect in equation (2). Furtherrnore, because an increase in $Y$ makes the blue edges hotter, thereby decreasing the periods, the effect of raising $Y$ is to translate the constant mass loci to the left in Figure 2.

Allowing for observational errors in determining $\phi_{31}$ (particularly for the $\omega$ Cen stars), and taking into account the standard deviation of the fit given by equation (2), we see from Figure 2 that the masses of the RRc stars seem to agree with the RR $d$ mass range noted above. It should be pointed out that the mass lines drawn in Figure 2 are really mean loci. Actually, even for fixed $Y$, the models of given mass spread out a bit in the diagram due to the fact that the same period can be attained with different luminosity and temperature. Thus the mass range encompassed by the $\mathrm{RR} c$ stars need not be quite so wide as Figure 2 suggests. This argument can be refined further by employing one more piece of information that we have regarding the $\omega$ Cen stars, namely their relative luminosities.

Let us choose an arbitrary "fiducial" star in $\omega$ Cen (it does not matter which one) and express the mass and luminosity of every other star in terms of this standard. Then, for any given star, we have from equation (1)

$$
\Delta \log L=0.105 \Delta \phi_{31}+1.814 \Delta \log M,
$$

where $\Delta$ has the sense: given star minus fiducial star. Notice that the $\log Y$ dependence in equation (1) is so weak that even if a range in $Y$ is allowed among the $\omega$ Cen stars (see below), the error in neglecting this term will still be small. Because 
TABLE 1

Parameters for $\omega$ Centauri RR $c$ Stars

\begin{tabular}{ccccc}
\hline \hline & \multicolumn{5}{c}{ CASE } \\
\cline { 2 - 5 } PARAMETER & \multicolumn{1}{c}{1} & \multicolumn{4}{c}{2} & \multicolumn{1}{c}{3} & 4 \\
\hline $\log M_{f} \ldots \ldots \ldots \ldots$. & -0.260 & -0.187 & -0.125 & -0.071 \\
$h_{2} \ldots \ldots \ldots \ldots \ldots \ldots$ & -0.884 & -0.732 & -0.603 & -0.490 \\
$\langle M\rangle \ldots \ldots \ldots \ldots \ldots$ & 0.48 & 0.57 & 0.66 & 0.75 \\
$M($ range) $\ldots \ldots \ldots \ldots$ & $0.42-0.57$ & $0.49-0.67$ & $0.57-0.78$ & $0.65-0.88$ \\
$\langle Y\rangle \ldots \ldots \ldots \ldots \ldots$ & 0.21 & 0.26 & 0.32 & 0.39 \\
$Y($ range $) \ldots \ldots \ldots \ldots$ & $0.19-0.23$ & $0.24-0.29$ & $0.29-0.35$ & $0.35-0.43$ \\
$\langle\log L\rangle \ldots \ldots \ldots \ldots$ & 1.58 & 1.71 & 1.81 & 1.90 \\
$\langle D\rangle \ldots \ldots \ldots \ldots \ldots$ & 13.9 & 14.2 & 14.4 & 14.7 \\
\hline
\end{tabular}

$\Delta \log L$ and $\Delta \Phi_{31}$ are given by the observations, equation (3) determines a value of $\Delta \log M$ for each star.

We now continue by fitting the reduced $\omega$ Cen sample with a relation of the form

$$
\phi_{31}=a \log P+b \Delta \log M+c .
$$

We obtain $a=5.83, b=-10.73, c=5.73$. Comparing with equation (2) one sees the excellent agreement between the observed and theoretical values of the coefficient $a$. We emphasize that this agreement was by no means mandated by our analysis (since eqs. [1] and [2] are independent) and is thus a vindication of the theoretical models.

However, it must also be noted that the observed coefficient $b=-10.73$ for the $\omega$ Cen stars is considerably more negative than the corresponding theoretical quantity in equation (2). The latter equation contains a strong helium dependence missing from equation (4) because the helium abundances of the $\omega$ Cen stars are not directly measurable. Suppose, however, that $\omega$ Cen comprises a range of $Y$ such that the helium abundance of a given star is linked to its mass, viz., $\log Y=h_{1}$ $\log M+h_{2}$. Constraining equation (2) with this relation, we obtain

$$
\begin{aligned}
\phi_{31}= & 6.013 \log P+\left(5.098 h_{1}-7.398\right) \Delta \log M \\
& +\left(5.098 h_{1}-7.398\right) \log M_{f}+5.098 h_{2}+7.571,
\end{aligned}
$$

in which we have written for convenience, $\log M=\log M_{f}$ $+\Delta \log M$, where $M_{f}$ is an arbitrary "fiducial" mass. Equation (5) represents a subset of the models for which $M$ and $Y$ are linked by the relation given just above.

We now proceed by forcing the best possible fit between the theoretical equation (5) and the "observational" equation (4) whose coefficients are already determined. The result is

$$
h_{1}=-0.63, \quad h_{2}=\left(10.60 \log M_{f}-1.75\right) / 5.098 .
$$

(Note that these numbers, obtained by least-squares fitting for the entire reduced $\omega$ Cen sample, are slightly different than what one would get by merely equating coefficients in eqs. [4] and [5].)

Thus, if there indeed exists a relationship between the masses of the $\omega$ Cen RRc stars and their helium abundances, there is enough information to determine its slope $h_{1}$ but not its zero point $h_{2}$. Further, note that because we have calculated $\Delta \log M$ for each $\omega$ Cen star (see eq. [3]), the selection of a value for $\log M_{f}$ not only determines $h_{2}$ from equation (6) but also fixes the mass of each $\operatorname{star}\left(\log M=\log M_{f}+\Delta \log M\right)$ and its helium abundance as well $\left(\log Y=-0.63 \log M+h_{2}\right)$. Thus the selection of a value for $\log M_{f}$ specifies both an average mass and average helium abundance for the $\omega$ Cen sample. In addition, once a mass and helium abundance have been calculated for each star, equation (1) yields a luminosity which, folded with the observed magnitudes (and neglecting reddening and bolometric corrections), gives an average distance modulus $\langle D\rangle$. Table 1 shows the results of four choices for the arbitrary parameter $\log M_{f}$, or equivalently the corresponding $h_{2}$ (see eq. [6] above).

\section{DISCUSSION}

We note first that the absolute (but not the relative) values of the numbers in Table 1 depend upon the linear pulsation theory blue edges. If these are hotter than we have calculated (e.g., due to the inclusion of convection or to different boundary conditions), the effect of this would be to translate the "helium rows" to the right with respect to the "mass rows," in Table 1, i.e., high masses would be indicated for more reasonable values of $Y$. Conversely, the effect of cooling the blue edges or of extending the overtone pulsation region redward would be to lower the mass at given $Y$.

Having said the above, we now emphasize that the present Table 1 shows that standard assumptions from pulsation theory yield, with reasonable helium abundances, masses for the $\omega$ Cen $R R c$ stars (e.g., case 2 in Table 1), which are in substantial agreement with the RRd masses. Whereas the values in Table 1 depend upon hydrodynamic light curves and linear blue edges, the RRd masses depend upon a quite different aspect of pulsation theory, namely, the calculation of periods. Thus standard pulsation theory yields a consistent result which is in clear disagreement with the much higher $R R$ Lyrae masses emerging, for example, from recent $H B$ evolution calculations, $M \geq 0.72 M_{\odot}$ (Lee, Demarque, and Zinn 1988), or from a recent study of $\omega$ Cen, $\langle M\rangle=0.73 M_{\odot}$ (Dickens 1989). A similar conclusion was reached by Cox (1988), based strictly on linear models.

Finally, it should be pointed out that analyses somewhat similar to ours have been done for years, but using $\log T_{e}$ as an observed variable instead of $\phi_{31}$. However, whereas the measurement of $\log T_{e}$ depends upon such quantities as blanketing and reddening, and such questions as the choice of a color index, the applicability of static atmosphere models and the definition of an equilibrium temperature (see, e.g., Lub 1987), the phase parameter $\phi_{31}$ may be measured to high accuracy given the combination of accurate observations and good phase coverage. Furthermore, the scheme we have presented can be checked by looking at RR $c$ stars in other clusters. For example, the RRc stars in an Oo II cluster like M15 ought to lie near the lower envelope in Figure 2, while Oo I RR $c$ stars (e.g., in IC 4499) should inhabit the upper envelope.

Unfortunately, much of the current observational data for clusters other than $\omega$ Cen seems to be marginal with regard to determining $\phi_{31}$ for the (low-amplitude) RRc stars. We urge that new, accurate CCD observations of globular cluster RR Lyrae stars be undertaken with this purpose in mind.

We wish to thank J. O. Petersen for kindly providing a table of $\omega$ Cen data, and the National Science Foundation for computing time on the Cray X-MP at the National Center for Supercomputing Applications. 


\section{REFERENCES}

Clement, C. M., Nemec, J. M. Robert, N., Wells, T., Dickens, R. J., and Bingham, E. A. 1986, A.J. 92, 825

Cox A. N. 1988, in IAU Colloquium 95, Second Conference on Faint Blue Stars, ed. A. G. Davis Phillip, D. S. Hayes, and J. W. Liebert (Schenectady: L. Davis), p. 161.

Dickens, R. J. 1989, in The Use of Pulsating Stars in Fundamental Problems of Astronomy, ed. E. G. Schmidt (Cambridge: Cambridge University Press), in press.

Kovacs, G., and Buchler, J. R. 1988, Ap. J., 324, 1026

Lee, Y.-W., Demarque, P., and Zinn, R. 1988, in IAU Colloquium 95, Second Conference on Faint Blue Stars, ed. A. G. Davis Phillip, D. S. Hayes, and J. W. Liebert (Schenectady: L. Davis), p. 137.
Lub, J. 1987, in Stellar Pulsation: A Memorial to John P. Cox, ed. A. N. Cox, W. M. Sparks, and S. Starrfield (Berlin: Springer), p. 218.

Petersen, J. O. 1984, Astr. Ap., 139, 496.

Simon, N. R. 1985, Ap. J., 299, 723.

. 1988a, in Pulsation and Mass Loss in Stars, ed. R. Stalio and L. A Willson (Dordrecht: D. Reidel), p. 27

. 1988b, Ap. J., 328, 747.

Simon, N. R., and Aikawa, T. 1986, Ap. J., 304, 249.

Norman R. Simon: Department of Physics and Astronomy, University of Nebraska, Lincoln, NE 68588-0111 